\title{
Endovaskuläre Schlaganfalltherapie
}

\section{Erfolge bei mechanischer Rekanalisation}

\author{
Die interventionelle endovaskuläre Schlaganfalltherapie macht Fortschritte. Erfolge bei der mecha- \\ nischen Rekanalisation verschlossener Hirnarterien mit neuen Thrombektomie-Systemen wurden jetzt \\ in zwei randomisierten Studien dokumentiert.
}

$\mathrm{T}$ herapie der Wahl beim akuten ischämischen Schlaganfall ist die intravenöse Thrombolyse innerhalb eines Zeitfensters von bis zu 4,5 Stunden nach Symptombeginn. Da diese Behandlung bei Verschlüssen von großen intrakraniellen Arterien wenig erfolgreich ist, kommen hier in jüngster Zeit zunehmend neue endovaskuläre Verfahren - sogenannte StentRetriever - zur mechanischen Rekanalisation zum Einsatz. Im Unterschied zu den echten Stents verbleiben die ihnen ähnelnden Retriever nicht dauerhaft in den Gefäßen.

\section{Stent-ähnlicher Retriever entfernt Thrombus}

$\mathrm{Zu}$ den neuen endovaskulären Verfahren zählen etwa der Trevo-Retriever des Unternehmens Stryker und das Thrombektomie-System Solitaire des Unternehmens Covidien. Beide Systeme ermöglichen es,

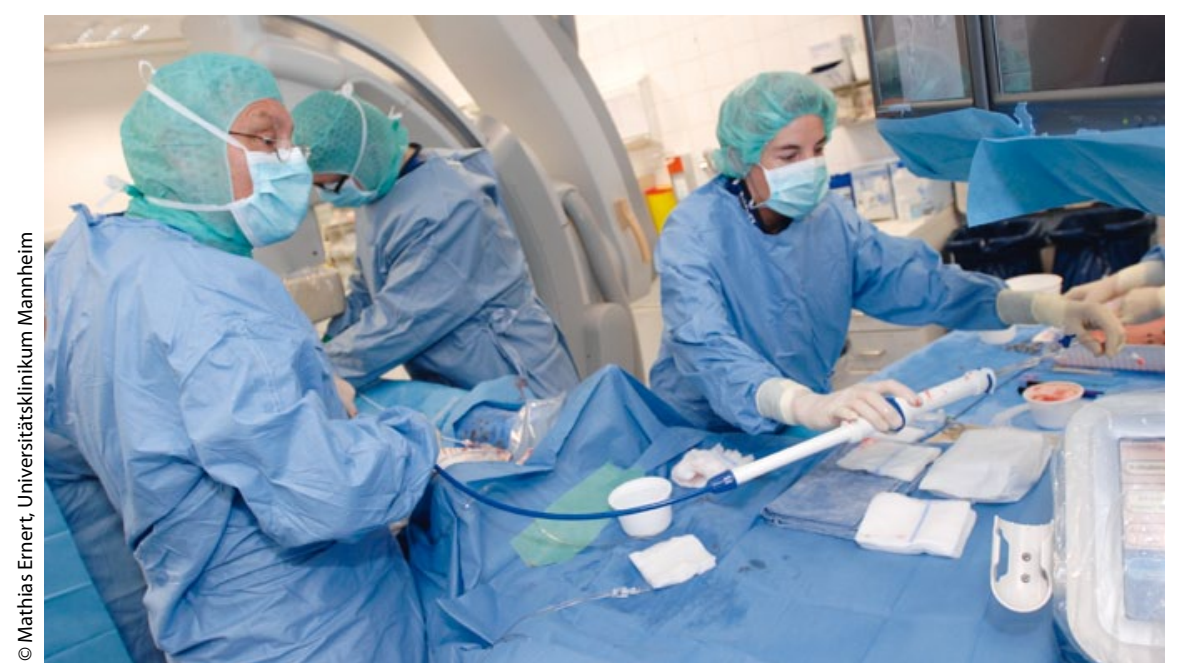

Stent-ähnliche Retriever könnten künftig auch bei Stent-Operationen hierzulande zum Einsatz kommen. in einem Gang den Blutfluss wiederherzustellen und das im feinen Drahtgeflecht des expandierten Stents festgehaltene Thrombusmaterial durch Rückzug des Retrievers zu entfernen.

Beide Systeme sind in einer randomisierten Studie (SWIFT und TREVO-2) jeweils mit einem seit 2004 in den USA von der FDA zugelassenen Thrombektomie-System (Merci) verglichen und die Ergebnisse in „The Lancet" publiziert worden. Das Solitaire-System wurde in der SWIFT-Studie untersucht, die im Juli 2011 vorzeitig gestoppt worden war. Teilnehmer waren 113 Schlaganfallpatienten, bei denen eine Thrombolyse entweder kontraindiziert oder zuvor erfolglos war.

Primärer Studienendpunkt war, einer Vorgabe der Zulassungsbehörden entsprechend, die erfolgreiche Gefäßrekanalisation ohne symptomatische intrakranielle Blutung. Dieses Ziel wurde mit dem So-

litaire-System bei $60,7 \%$ und mit dem konventionellen Verfahren bei $24,1 \%$ aller Patienten erreicht. Die Mortalitätsrate nach 90 Tagen war in der mit dem neuen Stent-Retriever behandelten Gruppe signifikant niedriger als in der Kontrollgruppe (17,2\% vs. $38,2 \%)$. Die Rate der Patienten mit einem als gut beurteilten neurologischen Status war zum gleichen Zeitpunkt signifikant höher (58,2\% vs. $33,3 \%)$.

\section{Höhere Erfolgsrate bei der Rekanalisation}

Erfreulich verlief auch der Vergleichstest mit dem Trevo-System in der TREVO-2Studie, an der 178 Patienten mit intrakraniellen Gefäßverschlüssen beteiligt waren. Primärer Endpunkt dieser Studie war die Rekanalisation der betroffenen Hirnarterien, definiert als TICI-Perfusionsgrad 2 oder höher. Gemessen an diesem Kriterium war die endovaskuläre Thrombektomie in der Trevo-Gruppe bei $86 \%$ und in der Merci-Gruppe bei $60 \%$ aller Patienten erfolgreich.

Der Anteil der Patienten mit als gut einzuschätzenden Aussichten auf ein unabhängiges Leben (modifizierter Rankin Score 0-2) war in der Trevo-Gruppe nach 90 Tagen signifikant höher, als in der mit dem Merci-System behandelten Vergleichsgruppe ( $40 \%$ v. $22 \%$ ). Die mit dem neuen Stent-Retriever erzielten Ergebnisse erfüllten die für den Nachweis sowohl der Nicht-Unterlegenheit als Überlegenheit festgelegten Kriterien.

Peter Overbeck

Nogueira RG et al. The Lancet 2012; 380:

1231-40

Saver JL et al. The Lancet 2012; 380: 1241-49 